\title{
Self-Organized Nanogratings for Large-Area Surface Plasmon Polariton Excitation and Surface-Enhanced Raman Spectroscopy Sensing
}

\author{
Matteo Barelli, Maria Caterina Giordano, Pietro Giuseppe Gucciardi, \\ and Francesco Buatier de Mongeot*
}

Cite This: ACS Appl. Nano Mater. 2020, 3, 8784-8793

Read Online

ACCESS | Lلll Metrics \& More | 回 Article Recommendations

Supporting Information

ABSTRACT: Surface plasmon polaritons (SPP) are exploited due to their intriguing properties for the fabrication and miniaturization of photonic circuits, for surface-enhanced spectroscopy and imaging beyond the diffraction limit. However, excitation of these plasmonic modes by direct illumination is forbidden by energy/momentum conservation rules. One strategy to overcome this limitation relies on diffraction gratings to match the wavevector of the incoming photons with that of propagating SPP excitations. The main limit of the approaches so far reported in the literature is that they rely on highly ordered diffraction gratings fabricated by means of demanding nanolithographic processes. In this work, we

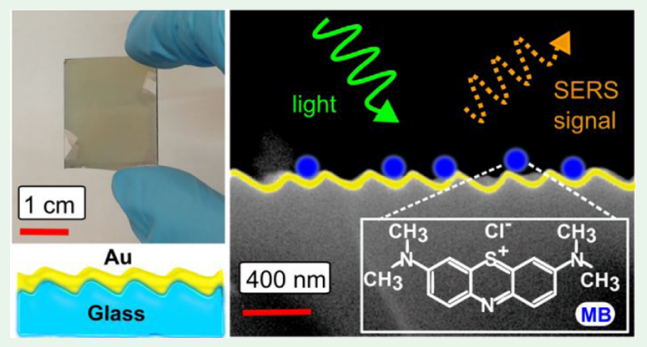
demonstrate that an innovative, fully self-organized method based on wrinklingassisted ion-beam sputtering can be exploited to fabricate large-area $\left(\mathrm{cm}^{2}\right.$ scale) nanorippled soda lime templates, which conformally support ultrathin Au films deposited by physical deposition. The self-organized patterns act as quasi-one-dimensional (1D) gratings characterized by a remarkably high spatial order, which properly matches the transverse photon coherence length. The gratings can thus enable the excitation of hybrid SPP modes confined at the Au/dielectric interfaces, with a resonant wavelength that can be tuned by modifying the grating period, photon incidence angle, or, potentially, the choice of the thin-film conductive material. Surface-enhanced Raman scattering experiments show promising gains in the range of $10^{3}$, which are competitive, even before a systematic optimization of the sample fabrication parameters, with state-of-the art lithographic systems, demonstrating the potential of such templates for a broad range of optoelectronic applications aiming at plasmon-enhanced photon harvesting for molecular or biosensing.

KEYWORDS: surface plasmon polaritons, surface-enhanced Raman scattering, self-organized plasmonics, large-area biosensors, self-organized nanopatterning

\section{INTRODUCTION}

In the last decades, the field of plasmonics witnessed an outstanding development because of the growing interest in the possibility to manipulate optical fields at the nanoscale, well below the diffraction limit, thus boosting a very broad range of optoelectronic applications. ${ }^{1-4}$ Different so-called plasmonic modes with diverse properties and resonant conditions are found in nature. ${ }^{5}$ In particular, surface plasmon polaritons (SPP) are electromagnetic modes propagating at the interface between a positive (e.g., a dielectric) and a negative permittivity medium due to resonant oscillations of free carriers. $^{5-10}$ Their propagating nature makes them suitable for waveguiding applications crucial for the development of photonic circuits and components, which are not enabled by localized surface plasmons (LSP) supported by subwavelength disconnected nanoparticles. ${ }^{5,6,11-16}$ SPP modes can be excited by electromagnetic radiation under selected wavevector coupling conditions, thus confining the photon energy into subwavelength volumes at the conductive/dielectric interface where the electromagnetic evanescent field is strongly enhanced. ${ }^{5,6,17}$ This peculiar property has recently allowed the highly sensitive near-field detection and imaging of surface plasmon modes with high subwavelength spatial resolution at the surface of atomic two-dimensional (2D) materials in scanning near-field optical microscopy $(\mathrm{SNOM})^{18-21}$ and molecular nanoimaging in tip-enhanced Raman scattering (TERS). ${ }^{22-25}$ In parallel, highly sensitive biosensing capabilities have been achieved in surface-enhanced spectroscopies such as surface-enhanced Raman spectroscopy (SERS), surface-enhanced coherent anti-Stokes Raman spectroscopy (SECARS), and metal-enhanced fluorescence (MEF), which

Received: June 8, 2020

Accepted: August 5, 2020

Published: August 5, 2020 


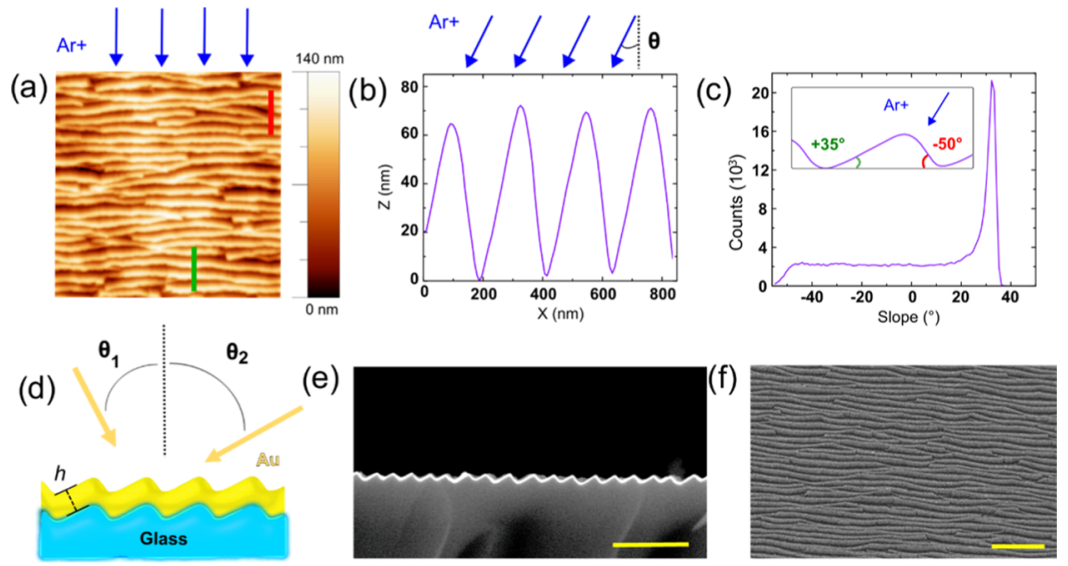

Figure 1. (a) Atomic force microscopy (AFM) topography of the rippled glass surface. The red scale bar corresponds to $800 \mathrm{~nm}$. (b) AFM line profile corresponding to the green line in (a). (c) Slope distribution histogram extracted by the AFM topography of (a). (d) Cross-sectional sketch of the two-step Au deposition process on the asymmetric sawtooth rippled profile of the soda lime glass surface. (e) Scanning electron microscopy (SEM) cross section and (f) top-view images of the Au-covered rippled surface, acquired in the backscattered electrons channel, with an acceleration voltage of $15 \mathrm{kV}$. The yellow bars correspond to $800 \mathrm{~nm}$ (e) and $2 \mu \mathrm{m}$ (f).

provide signal gain of several orders of magnitude over their conventional counterparts. ${ }^{4,26-30}$ Moreover, traveling SPPs have been exploited to perform remotely excited SERS (RESERS), a technique where excitation and SERS signal collection are spatially displaced, remarkably reducing the fluorescence background signal originating from diffractionlimited excitation volumes. ${ }^{31,32}$ Several applications have been recently developed, such as optical refractive index sensor devices, already available in the market, which exploit the sensitivity of the SPP resonance to the refractive index of the surrounding dielectric medium. ${ }^{33-35}$ An optical nanoimprint technique has been developed exploiting interfering SPP waves excited by the scattering due to surface defects of focused femtosecond laser pulses, leading to the creation of periodic ripple-like structures (laser-induced periodic surface structures-LIPSSs) thorough ablation over micrometer-scale areas. $^{36-38}$ Recently, it has been proved that the technique can be scaled to large $\mathrm{cm}^{2}$ areas even if it remains intrinsically limited to the patterning of absorptive semiconducting and metallic surfaces. ${ }^{39}$ Alternatively, nonradiative plasmon decay generating hot electrons into the conductive material can be exploited to enable direct plasmon-enhanced photocataly$\operatorname{sis}^{40,41}$ and/or hot carrier injection into semiconductors, effectively extending the operating range of photonic devices beyond their band-gap-limited absorption. ${ }^{42,43}$ However, photon-SPP coupling is not allowed by direct illumination because of energy and momentum conservation selection rules; thus, particular strategies have to be adopted, such as EM coupling via subwavelength probes or diffraction gratings, which are able to modulate the wavevector of the incoming photons. ${ }^{5,6}$ In particular, diffraction gratings characterized by a very high spatial coherence are typically fabricated via topdown lithographic fabrication methods that, however, can limit the perspective of large-scale, low-cost applications. ${ }^{27-29,44-47}$

In this work, we demonstrate that a cheap, transparent nanorippled soda lime glass template, prepared by an innovative, fully self-organized ( $\mathrm{SO}$ ) fabrication method based on wrinkling-enhanced ion-beam sputtering, ${ }^{48}$ can be used as large-area support for a thin Au film conformally grown on top of it by thermal vacuum deposition, in an insulatormetal-insulator (IMI) configuration. The resulting semitransparent, subwavelength, quasi-one-dimensional (1D) gra- ting endowed with linearly graded periodicity enables the excitation of a tunable hybrid surface plasmon polariton mode. Based on our knowledge, this is the first example of fully selforganized gratings promoting the coherent excitation of SPP modes over large area.

The potential of these large-area plasmonic meta-surfaces for highly sensitive biosensing has been demonstrated in SERS, showing strong enhancement of the Raman signal promoted by SPP modes. Remarkable SERS gain values in the range of $10^{3}$, competitive with the figures of lithographic-made systems ${ }^{49,50}$ as well as with alternative self-organized approaches, ${ }^{51-54}$ are found for a pump laser wavelength of $785 \mathrm{~nm}$. Indeed, this makes the sample appealing for molecular sensing, also considering that a systematic optimization of the sample fabrication parameters, which is discussed but it is beyond the scope of this work, could easily further improve these SERS gain values. Finally, we demonstrate how the SERS gain is strongly correlated with the electric field enhancement produced by the excitation of SPP modes by comparing SERS and co-localized microextinction measurements. These self-organized gratings represent a very versatile platform, potentially allowing the broad-band tuning of the SPP mode from the near-UV to the far-IR by simply selecting the conductive layer grown on top of the template, making them interesting for a broad range of optoelectronic applications.

\section{METHODS}

2.1. Sample Fabrication. A soda lime glass $\left(2.5 \times 2.5 \times 0.2 \mathrm{~cm}^{3}\right)$ is heated up to $680 \mathrm{~K}$ and irradiated with a defocused ion beam $\left(\mathrm{Ar}^{+}\right)$ with a low energy of $800 \mathrm{eV}$ at the incidence angle $\theta=30^{\circ}$ (Figure lb) with respect to the sample surface normal direction. Positive charge buildup on the glass surface is prevented during the ion irradiation process by stimulating thermionic electron emission from a tungsten filament negatively biased at $V_{\text {bias }}=-13 \mathrm{~V}$ with respect to the glass sample. For ion irradiation prolonged for $1800 \mathrm{~s}$ at the pressure of $4 \times 10^{-4} \mathrm{mbar}$ (i.e., ion fluence of $1.4 \times 10^{19} \mathrm{ions} / \mathrm{cm}^{2}$ ), highly ordered faceted nanopatterns evolve on the glass surface, showing a steep, asymmetric faceted sawtooth profile. After the nanorippled pattern is formed on the sample surface, thermal $\mathrm{Au}$ deposition is performed on the rippled template at the pressure of about $10^{-5} \mathrm{mbar}$ in a two-step process, as described in the Section 3. The process is designed to grow an Au thin film of approximately homogeneous thickness on the slope-modulated rippled glass surface. 
2.2. Morphology Characterization. The self-organized rippled glass morphology is characterized by means of an atomic force microscope (Nanosurf S Mobile). The average periodicity, line profiles, and slope of the nanoripples are extracted by means of WSxM and Gwyddion software from the statistical analysis of AFM measurements. ${ }^{55,56}$ Top-view backscattered electron SEM images are acquired by means of a thermionic Hitachi VP-SEM SU3500.

2.3. Optical Characterization. Normal incidence polarized transmission measurements are performed in both transverse electric (TE, electric field parallel to the grating grooves) and transverse magnetic (TM, electric field orthogonal to the grating grooves) configuration by fiber-coupling the radiation emitted by a compensated deuterium halogen lamp (DH-2000-BAL, Mikropak) to a linear polarizer, illuminating the sample from the bare glass side. The beam spot diameter is about $1 \mathrm{~mm}$. The signal is then coaxially collected by a high-resolution solid-state spectrometer (HR4000, Ocean Optics). Non-normal incidence transmission measurements are performed in TM polarization by placing the sample on a stage which provides a tilting movement. All of the transmission spectra are normalized to the optical transmittance of a bare glass reference substrate tilted at the corresponding sample angle.

2.4. SERS and Co-localized Extinction Measurements. Samples (rippled $\mathrm{Au} /$ soda lime glass and reference flat $\mathrm{Au}$ thin film on bare soda lime glass) are immersed in a $10^{-4} \mathrm{M}$ methylene blue (MB) solution for an hour and then rinsed in deionized water for 10 min. Polarized SERS and optical microextinction measurements are performed by means of a Horiba XploRA Nano. For SERS, pump lasers at 638 and $785 \mathrm{~nm}$ wavelengths are employed, using a 100X objective for the excitation of a diffraction-limited area. Different sample positions, a few millimeters away from each other, are probed by averaging multiple SERS measurements distanced by some $\mu \mathrm{m}$. Detection is carried out in backscattering. Microextinction measurements have been co-locally performed at corresponding sample coordinates by switching the source to a tungsten lamp illuminating the sample from below and to a $10 \times$ objective for signal collection.

\section{RESULTS AND DISCUSSION}

A defocused $\mathrm{Ar}^{+}$-ion beam at low energy $(800 \mathrm{eV})$ irradiates a soda lime glass sample at an incident angle $\theta=30^{\circ}$ with respect to the surface normal, as shown in Figure 1a,b. The sample temperature is fixed at about $680 \mathrm{~K}$ during the $\mathrm{Ar}^{+}$ bombardment (see Section 2.1). The ion-beam sputtering process induces a self-organized quasi-1D nanorippled morphology all over the glass surface, with propagation vector parallel to the incoming ion-beam direction (Figure 1a). The ripples length extends over several micrometers, and the pattern shows a noteworthy degree of long-range order. The glass nanostructures are characterized by a high aspect ratio asymmetric sawtooth profile, with vertical dynamic range of about $75 \mathrm{~nm}$ (Figure $1 \mathrm{~b}$ ) and periodicity of approximately 200 $\mathrm{nm}$, assessed by the two-dimensional self-correlation function of the AFM topography (see Figure S1). The ripple sides opposed to the incoming ion-beam direction develop wider facets with a narrow slope distribution sharply peaked at about $+35^{\circ}$ (Figure 1c). Conversely, the sides directly exposed to the $\mathrm{Ar}^{+}$beam develop narrower facets with a broad slope distribution weakly peaked at about $-50^{\circ}$ (Figure 1c). Moreover, we engineered the IBS process to produce a sample with a graded periodicity along its length. We achieved this by exploiting an ion dose gradient along the projection of the Ar+ beam, originating from the increasing sample-source distance at the tilted incidence angles employed here, and from the gaussian intensity distribution of the ion beam. Considering a periodicity of about $\Lambda=200 \mathrm{~nm}$ in the central area of the glass template (Figure 1a), we achieve in this way a modulation of the grating periodicity of about $\pm 15 \%$ moving from the top to the bottom of the sample along the projection of the ion beam (see Figure S2). It is worth to underline, as described in a recent study by some of the authors, ${ }^{48}$ that the nanopattern formation kinetics is strongly enhanced by a solid-state wrinkling instability, which is activated when the substrate temperature is raised near the glass-transition threshold and is concurrently exposed to irradiation with an Ar+-ion beam. Under such conditions, a compressive stress builds up in a thin surface skin layer partially depleted of alkali atoms; stress release takes place by surface buckling, provided enhanced mass transport in the near-surface region is made possible by the reduction in glass viscosity. ${ }^{48}$ We highlight that when ion irradiation is performed at reduced temperatures for which mass transport is strongly reduced, one does not observe the formation of the high aspect ratio faceted wrinkles but rather the low aspect ratio ripples conventionally found after ionbeam sputtering of insulating and semiconductor substrates at room temperature. ${ }^{57-59}$

As demonstrated by the authors in recent studies ${ }^{60-62}$ such faceted, high aspect ratio, ordered rippled patterns are the ideal template for maskless confinement of large-area, self-organized arrays of disconnected uniaxial plasmonic nanostructures. The possibility to control tilt and morphology of the nanostructures is an interesting feature for a wide range of applications that exploit tunable dipolar and hybrid localized plasmonic modes. Here, instead, we propose a novel self-organized fabrication protocol to produce periodically corrugated continuous $\mathrm{Au}$ thin films of uniform thickness, conformally supported on the linearly graded glass diffraction grating that sustain propagating surface plasmon polariton (SPP) modes with tunable response.

Given the asymmetric faceted profile of the glass template, a conventional thermal deposition process at normal incidence would result in a Au film with nonuniform thickness, evaluated along the local surface normal. To overcome this limitation, we developed a dual-step off-normal thermal deposition procedure capable of achieving growth of an $\mathrm{Au}$ film with uniform thickness on the two opposite facets. The first deposition step is performed at the angle $\theta_{1}=-15^{\circ}$ with respect to the sample surface normal (negative angles corresponding to an anticlockwise rotation), over the side of the ripple profile opposing the ion beam during the nanostructuring step (Figure 1d). Under this condition, the Au beam forms a $20^{\circ}$ angle with respect to the local surface normal of the "wide" facets. At the same time, an angle of $65^{\circ}$ is formed between the Au beam and the local surface normal to the "steep" facets. The Au thickness deposited during the first deposition step on the left side of the ripple can be estimated as $h_{\text {left-1 }}=h_{0} \times \cos 20^{\circ}$, while on the right side, the thickness is $h_{\text {right-1 }}=h_{0} \times \cos 65^{\circ}$, where $h_{0}$ is the equivalent $\mathrm{Au}$ thickness on a flat substrate. During the experiment, $h_{0}$ is the parameter that is measured, by means of a quartz microbalance. The second $\mathrm{Au}$ deposition step is performed at the angle $\theta_{2}=+30^{\circ}$ with respect to the sample normal (positive angles correspond to a clockwise rotation), over the side of the ripple profile directly facing the ion beam during the nanopatterning step (Figure 1d). Now, the $\mathrm{Au}$ beam forms a $20^{\circ}$ angle with respect to the steep facets and a $65^{\circ}$ angle with respect to the wide facets local surface normal; the situation is now opposite compared to $\theta_{1}$ conditions and $h_{\text {left-2 }}=h_{0} \times \cos 65^{\circ}$ while $h_{\text {right-2 }}=h_{0} \times \cos 20^{\circ}$. To grow an approximately uniform $\mathrm{Au}$ layer over the asymmetric slope modulate ripple profile, it is sufficient to impose a desired thickness $h$ and solve one of the following equations for $h_{0}$ 


$$
\begin{aligned}
& h_{\text {left }-1}+h_{\text {left }-2}=h_{0} \times \cos 20^{\circ}+h_{0} \times \cos 65^{\circ}=h \\
& h_{\text {right }-1}+h_{\text {right-2 }}=h_{0} \times \cos 65^{\circ}+h_{0} \times \cos 20^{\circ}=h
\end{aligned}
$$

In view of SPP excitation, we select $h=25 \mathrm{~nm}$ since it allows us to combine optical transparency and electrical percolation of the polycrystalline thin film, thus enabling standard far-field transmission spectroscopy for probing SPP propagation in the continuous $\mathrm{Au}$ layer. As shown in the scanning electron microscopy (SEM) cross-sectional image of the sample after metal deposition (Figure 1e), one can clearly identify the presence of the Au film with thickness $h=25 \pm 0.5 \mathrm{~nm}$ (Figure 1d), which conformally follows the profile of the glass template. Such a rippled air/Au thin film/glass template can be considered as an IMI (insulator-metal-insulator) structure with slanted facets. We stress that such asymmetric, so-called slanted (or blazed), quasi-1D gratings cannot be fabricated by top-down techniques unless complex grayscale lithography processes are adopted.

For the excitation of the propagating plasmon, it is necessary that proper coupling of light momentum takes place at the metal/dielectric interface, mediated by transfer of the grating wavevector $k_{\mathrm{G}}=n \frac{2 \pi}{\Lambda}$ (see refs 5, 6 and the references therein). The latter process additionally requires that the spatial coherence of the grating pattern (Figure 1f) is comparable to the transverse coherence length $L_{\mathrm{t}}=0.16 \frac{\lambda D}{\delta}=9.6 \mu \mathrm{m}$ of the exciting thermal light beam at a nominal wavelength $\lambda=600$ $\mathrm{nm}$, collimated by an optical fiber with a core diameter $\delta=1$ $\mathrm{mm}$ placed at a distance $D=0.1 \mathrm{~m}$ from the grating. Indeed, the two-dimensional self-correlation analysis of the sample AFM topography (see Figure S3) demonstrates the visibility of coherent oscillations at the grating periodicity $\Lambda \approx 200 \mathrm{~nm}$ even for lateral translations of the order of $10 \mu \mathrm{m}$, which are comparable to the typical transverse coherence length $L_{t}$ of incoming photons. This striking degree of long-range morphological order, considering the fully self-organized nature of our diffraction nanogratings, enables the possibility to investigate them as large-area launchers of SPP modes at optical frequencies.

Normal incidence linearly polarized optical transmission spectra are shown in Figure 2a.

For TE polarization (Figure 2a, dotted black curve), the electric field oscillates parallel to the diffraction grating grooves (ripples long axis); in the considered spectral range, the sample transmission curve resembles the one of a Au flat thin film of equivalent thickness ${ }^{63,64}$ (Figures $2 \mathrm{~b}$ and S4). For TM polarization (Figure 2a, continuous red curve), the electric field oscillates orthogonally to the diffraction grating grooves (ripples short axis) and a strong dichroism is observed as transmission shows a broad minimum centered at the wavelength $\lambda$ of about $660 \mathrm{~nm}$. This transmission dip (corresponding to a maximum in the extinction spectrum) is attributed to the excitation of a propagating SPP mode along the continuous rippled $\mathrm{Au}$ film enabled by the momentum exchange mediated by photon-grating interaction, in accordance with previous works. ${ }^{27,29,35,44}$

In Figure 2c, we demonstrate that the SPP resonant wavelength, measured in the transmission spectra under TM polarization at normal incidence, can be tuned by varying the grating periodicity at different sample coordinates along our linearly graded nanogratings. By statistical analysis of the $2 \mathrm{D}$ self-correlation functions of multiple AFM topographical
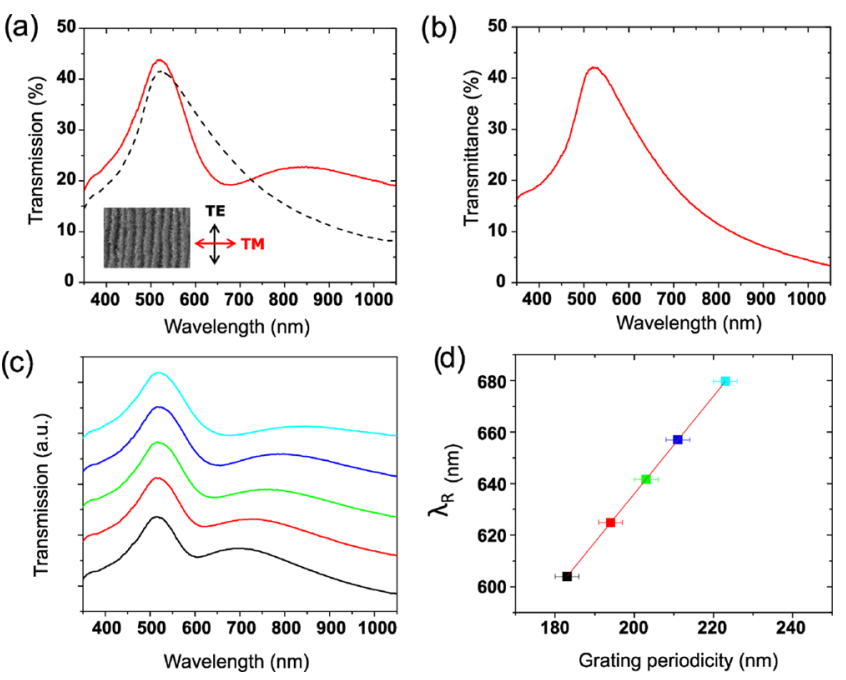

Figure 2. (a) Normal incidence transmission measurements as a function of polarization. TM polarization corresponds to the continuous red curve, while TE polarization corresponds to the black dashed curve, as shown in the inset. (b) Polarizationindependent transmission spectrum of a flat $25 \mathrm{~nm}$ thick $\mathrm{Au}$ film on bare glass. (c) TM normal incidence transmission spectra acquired for different periodicities of the Au/soda lime blazed grating; $183 \mathrm{~nm}$ (black curve), $194 \mathrm{~nm}$ (red curve), $203 \mathrm{~nm}$ (green curve), $211 \mathrm{~nm}$ (blue curve), $223 \mathrm{~nm}$ (cyan curve). (d) Plot of resonant wavelength vs. grating periodicity. The error bars on the grating periodicity are 3 $\mathrm{nm}$ wide. All of the transmission spectra shown in this panel are normalized to the one of a flat bare glass.

images acquired in proximity of the different optical spot coordinates, we can associate the average grating periodicity co-localized with the optical transmission spectra. In Figure 2d, the wavelength of the transmission dip minimum of each spectrum is plotted against the corresponding grating periodicity. The SPP resonant wavelength red-shifts linearly and monotonically starting from the smaller considered periodicity $(\Lambda=183 \mathrm{~nm}$; black curve in Figure 2c) to the bigger one $(\Lambda=223 \mathrm{~nm}$; cyan curve in Figure 2c). Such a monotonic red shift of the SPP wavelength with increasing grating periodicity $\Lambda$ is to be expected since the momentum exchange $k_{\mathrm{G}}=n \frac{2 \pi}{\Lambda}$, mediated by photon-grating interaction, depends reciprocally on the period ( $n$ is an integer either positive or negative; a more detailed discussion follows)., The broad-band nature of the mode (full width at half maximum of about $100 \mathrm{~nm}$ ) is here mainly attributed to electron scattering at the $\mathrm{Au}$ grain boundaries ${ }^{65}$ of the polycrystalline film. Compared to the optical behavior of similar thin films supported by lithographic gratings, ${ }^{29}$ we observe slightly broader SPP resonances due to the statistical distribution of our self-organized grating grooves periodicity $\Lambda$.

To further investigate and strengthen the attribution of the observed optical extinction loss to the excitation of an SPP mode, transmission measurements as a function of the sample tilt relative to the light beam were performed. The sample is illuminated from the glass side, and the signal is coaxially collected in extinction configuration in TM polarization. The sample is tilted, forming an angle $\theta$ between the incident light beam and the surface normal (Figure $3 \mathrm{a}$ ). The sample rotation is performed counterclockwise, that is, reducing the angle formed by the incident light beam and the local surface normal of the wide facets of the rippled $\mathrm{Au}$ /glass template (Figure 3a). 
(a)

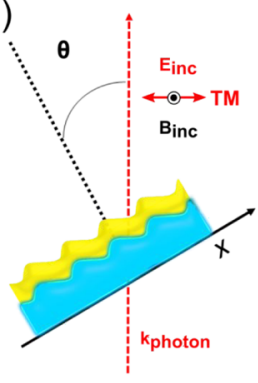

(b)

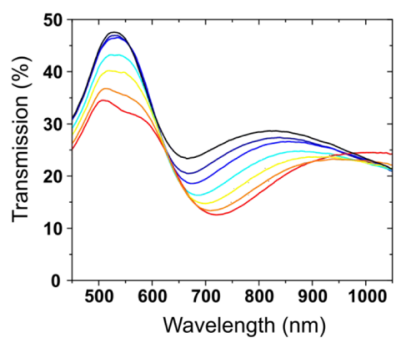

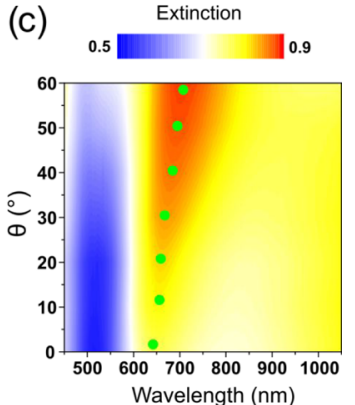

Figure 3. (a) Sketch of the tilted transmission $(T)$ measurements configuration. The red arrow $k_{\text {photon }}$ represents the light beam incoming from the glass side. $\theta$ is the angle formed between the light beam and the normal to the flat sample surface. The $x$ arrow defines the axis along the metal dielectric interfaces. The inset shows the direction of oscillation of the incoming electric field $E_{\text {inc }}$ (red arrow) and the out-of-plane magnetic field $B_{\text {inc }}$ (black dot). (b) TM transmission measurements as a function of $\theta: 0^{\circ}$ (black curve), $10^{\circ}$ (royal blue curve), $20^{\circ}$ (blue curve), $30^{\circ}$ (cyan curve), $40^{\circ}$ (yellow curve), $50^{\circ}$ (orange curve), and $60^{\circ}$ (red curve). (c) Extinction maps (calculated from the transmission spectra as $\left.\frac{100-T(\%)}{100}\right)$ as a function of both wavelength and tilt angle $\theta$. The uncertainty on the tilt angle $\theta$ is $\pm 1^{\circ}$.

In Figure $3 b$, transmission spectra are plotted for different sample tilts, increasing in $10^{\circ}$ steps from 0 to $60^{\circ}$.

The transmission dips red-shifts monotonically with increasing sample tilt, confirming the dispersive nature of the observed propagating SPP mode. For the highest tilt $\theta=60^{\circ}$ (red curve in Figure $3 \mathrm{~b}$ ), an additional, weak relative transmission minimum is observed at about $\lambda=540 \mathrm{~nm}$. To better visualize the experimental trend, in Figure $3 c$, the wavelength-dependent extinction map (computed as $\frac{100-T(\%)}{100}$ ) is plotted against both wavelength $\lambda$ and sample tilt $\theta$. The nondispersive blueish extinction band centered at about 530 $\mathrm{nm}$ is clearly associated with the spectral edge at the onset of $\mathrm{Au}$ interband transitions, while the strong and dispersive red band describes the SPP mode, which intensifies and broadens with increasing sample tilt.

The monotonic red shift of the SPP resonance with increasing sample tilt (i.e., with increasing projection of photon wavevector $k_{\mathrm{ph}}$ in the positive $x$-direction) suggests the unidirectional counter-propagating nature of the SPP mode with respect to the incident light beam. This trend can be reconciled, considering that the grating allows us to exchange momentum $k_{\mathrm{G}}=n \frac{2 \pi}{\Lambda}$ in discrete units, and the effective wavevector component $k_{x}$ along the $x$ axis can be derived according to the well-known relation: $k_{x}=\left|k_{\mathrm{ph}}\right| \sin \theta+n \frac{2 \pi}{\Lambda}$, where $k_{\mathrm{ph}}$ is the modulus of photon wavevector, $\theta$ is the angle formed by the light beam with the normal to the flat sample surface (Figure $3 \mathrm{a}$ ), $\Lambda$ is the periodicity of the $\mathrm{Au} /$ soda lime grating in the region of the optical spot, and $n$ is an integer, either positive or negative. In particular, given the wavevector of the photon in correspondence to the SPP mode and the periodicity $\Lambda$ of the grating, one observes that for negative $n$ values, $k_{x}$ is negative and decreases in modulus for increasing values of $\theta$.

To better highlight the observed trend of the dispersion relation of the surface plasmon, in Figure $4 a$, we plot jointly the experimental SPP energy $\hbar \omega_{\text {SPP }}$ and the theoretical value derived for a flat $\mathrm{Au}$ slab as a function of the in-plane wavevector using the conventional expression $k_{\mathrm{SPP}}=\frac{\omega_{\mathrm{SPP}}}{c} \sqrt{\frac{\varepsilon_{1} \varepsilon_{2}}{\varepsilon_{1}+\varepsilon_{2}}}$, where $\varepsilon_{1}$ is the real part of the dielectric function of a thin flat $\mathrm{Au}$ film ${ }^{63}$ and $\varepsilon_{2}$ is the real part of the effective dielectric function of the corrugated glass supporting the $\mathrm{Au}$ slab. The plotted SPP branch-light blue line- (a)
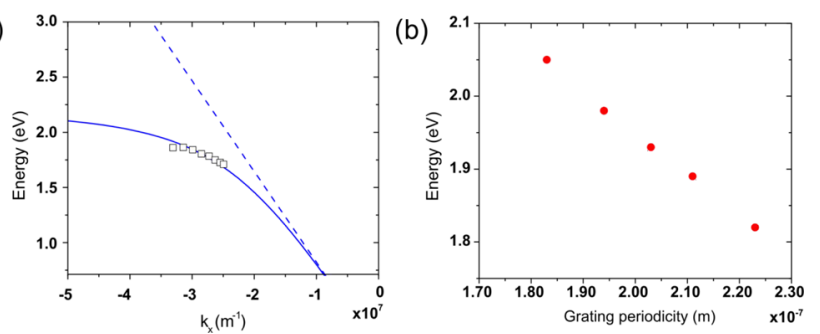

Figure 4. (a) Dispersion relation of the short-range antisymmetric SPP mode $\hbar \omega_{\text {SPP }}$ vs $k_{x}$ propagating at the $\mathrm{Au} /$ dielectric interface (solid blue curve) and light line of a photon traveling in the dielectric effective medium (dashed blue curve). Experimental data are plotted as squares. (b) Plot of resonant energy $\hbar \omega_{\mathrm{SPP}}$ of the short-range hybrid SPP mode at normal incidence vs. grating periodicity.

corresponds to the counter-propagation of the SPP confined at the $\mathrm{Au}$-glass interface with effective dielectric refractive index $n_{2}=2.3$ (the straight dashed line is the dispersion relation of light in the dielectric medium). The experimental values of the SPP energy $\hbar \omega_{\text {SPP }}$, associated with the transmission dips of Figure $3 \mathrm{~b}$, are plotted in terms of $k_{x}=\left|k_{\mathrm{ph}}\right| \sin \theta+n \frac{2 \pi}{\Lambda}$ assuming $n=-1$, i.e., incoming photons with positive momentum $k_{x}$ couple with an SPP with negative $k_{x}$, via exchange of grating coupled negative momentum $k_{\mathrm{G},-1}=-\frac{2 \pi}{\Lambda}$.

We stress that the comparison of Figure 4, though simplified and based on an effective medium approximation, allows us to describe the general trend of SPP dispersion when the Au film is so thin $(h=25 \mathrm{~nm}$ ) that coupling between plasmon polaritons propagating at the two interfaces of the slab cannot be neglected. This leads to a hybridization of the SPP modes of a thick metal slab into an antisymmetric mode (out-of-phase matching of electric fields at the two sides of the metal film, also called short-range hybrid mode), which is red-shifted compared to the single-interface metal/glass SPP, and into a symmetric mode (in-phase matching of electric fields at the two sides of the metal film, also called long-range hybrid mode), which is blue-shifted compared to the single-interface air/metal SPP. ${ }^{66-69}$ The best match with the experimental data (Figure $3 \mathrm{~b}, \mathrm{c}$ ) corresponding to the low energy antisymmetric mode is found for an effective refractive index of the corrugated glass $/ \mathrm{Au}$ slab $n_{2}=2.3$, a considerably higher value compared to the typical $n=1.5$ of glass, which would be expected in the thick $\mathrm{Au}$ film limit. In our experimental 
(a)

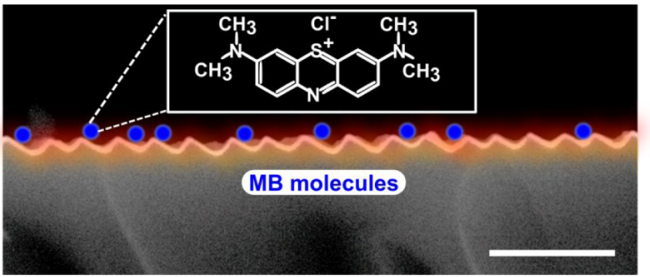

(b)

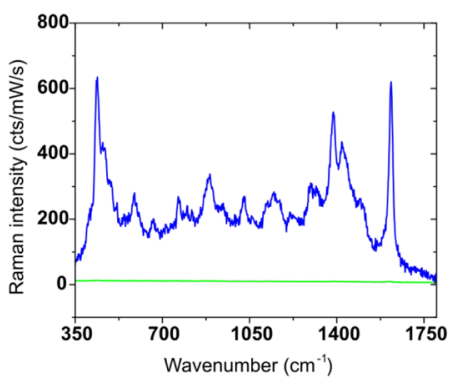

(d)

(f)

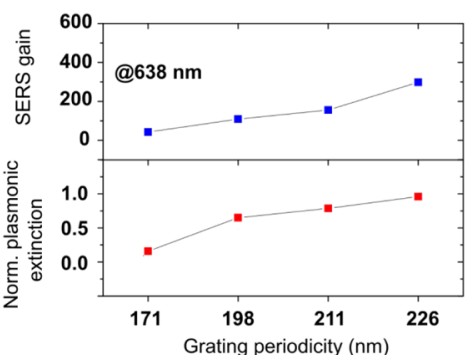

(c)

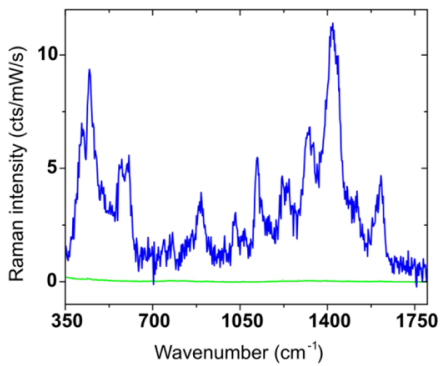

(e)

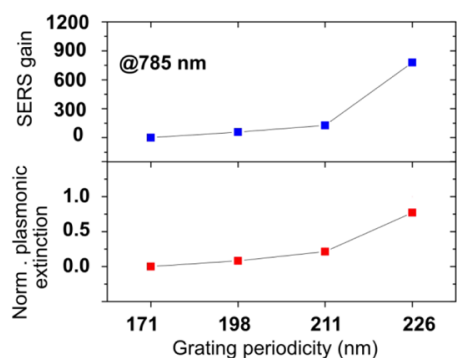

Figure 5. (a) Rendered version of the resonant nanograting SEM cross section with methylene blue molecules absorbed on its surface. The white scale bar is $800 \mathrm{~nm}$ long. (b, c) Sample SERS spectra (blue traces) and reference flat Au film Raman signal (green traces) for $638 \mathrm{and} 785 \mathrm{~nm}$ pump lasers, respectively. (d, e) SERS gain of MB molecules signal with 638 and $785 \mathrm{~nm}$ pump lasers, respectively, for different grating periodicity. The error on the SERS gain values is esteemed to be about 5\%. Error bars are not drawn because they merge into the data symbols. (f, g) Plasmonic extinction values at 638 and $785 \mathrm{~nm}$, respectively, as a function of grating periodicity, derived from Raman co-localized transmission measurements (see Figure S5).

configuration, the high-energy symmetric hybrid SPP mode is not observable since it is strongly damped by the Au s-d interband transitions, which are active below $520 \mathrm{~nm}$.

In Figure $4 \mathrm{~b}$, instead, we highlight the possibility to modify the experimental SPP plasmon energy $\hbar \omega_{\text {SPP }}$ by just varying the grating periodicity $\Lambda$ (see Figure $2 \mathrm{~d}$ ), when excitation takes place at a constant incidence angle (normal incidence, $\theta=0^{\circ}$ ). The theoretical value of the SPP energy $\hbar \omega_{\mathrm{SPP}}=\hbar c k_{\mathrm{SPP}} \sqrt{\frac{\varepsilon_{1}+\varepsilon_{2}}{\varepsilon_{1} \varepsilon_{2}}} \quad$ is $\quad$ e v a lu a t e d for $k_{\mathrm{SPP}}=k_{x,-1}=\left|k_{\mathrm{ph}}\right| \sin \theta+n \frac{2 \pi}{\Lambda}$ in correspondence to the diffracted order $n=-1$ and $\theta=0^{\circ}$. Matching between experimental and theoretical values, when the grating period progressively increases from 183 to $223 \mathrm{~nm}$, is achieved by optimizing the only free parameter, the effective refractive index of the undulated dielectric layer $n_{2}$ supporting the $\mathrm{Au}$ film, in a narrow range $(2.28 \pm 0.08)$.

Similar to the case of localized plasmon resonances supported by nanoparticles, the incoming electric field is strongly enhanced and confined near the dielectric interface of the metal film supporting an SPP. ${ }^{5,6}$ In particular, previous works investigated the near-field distribution of resonant hybrid SPP modes over periodically corrugated thin films. ${ }^{69-73}$ In our experiment (Au thickness $h=25 \mathrm{~nm}$ ), the evanescent field of the short-range hybrid SPP is able to reach the outer half layer occupied by air, where it can couple with molecular probes located therein. ${ }^{66-68}$ With this in mind, we recurred to SERS/microextinction measurements to probe the SPP nearfield enhancement on the rippled $\mathrm{Au} /$ soda lime blazed grating. Methylene blue (MB) was used as a probe molecule (Section 2.4). Different sample positions a few millimeters apart from each other have been probed to verify the dependency of the SERS signal from the varying periodicities of our graded nanogratings (Figure 2c). For each considered sample position, TM-polarized SERS and co-localized optical microextinction measurements are performed (Section 2.4). As a reference, we acquired the Raman signal of $\mathrm{MB}$ absorbed under the same experimental conditions on a flat $\mathrm{Au}$ film with a thickness of $150 \mathrm{~nm}$ (the SERS signal-green line-is negligible when plotted in linear scale). This allows us to estimate the SERS gain of our rippled substrate, defined as the ratio between the intensity of the peak at $1625 \mathrm{~cm}^{-1}$ measured on the rippled sample (e.g., blue curves in Figure 5b,c) and on the flat Au film (green curves in Figure $5 b, c$ ), normalized to the laser power and integration time. ${ }^{51}$ Similar SERS gain values are found by considering the signal of $\mathrm{MB}$ absorbed on glass or $\mathrm{CaF}_{2}$ substrates as a reference. ${ }^{74}$ The Raman signal intensity of $\mathrm{MB}$ on glass/CaF2 closely matches that on flat gold at both 785 and $633 \mathrm{~nm}$ pump lasers. The so-defined SERS gain, plotted as a function of the grating periodicity in each considered position for the 638 and $785 \mathrm{~nm}$ pump lasers, 
shows a monotonic increasing trend, as reported in Figure $5 \mathrm{~d}$,e, respectively.

As previously discussed, the SPP resonance spectrally shifts when moving at different sample coordinates, due to a spatial dependence of the SO grating periodicity (Figure 2c). It is thus possible to nicely correlate the intensity of the plasmonic extinction strength at the pump lasers frequency, which is responsible for the SERS near-field enhancement, with the colocalized measured SERS gains. After background subtraction, the extinction values at the pump laser wavelengths of 638 and $785 \mathrm{~nm}$ are then plotted as a function of different sample positions in Figure 5f,g, respectively (see Figure S5 for details). Remarkably, the intensities of TM extinction and co-localized SERS gain are very strongly correlated for both pump laser wavelengths as a function of sample coordinates, thus confirming that the observed SERS signal is indeed matched with the strength of the SPP-induced electric near-field enhancement.

The SERS gain with the $638 \mathrm{~nm}$ laser turns out to be of the order of $3 \times 10^{2}$. It must be noted that at $638 \mathrm{~nm}$ an electronic transition in MB molecules is excited. In this case, surfaceenhanced resonant Raman (SERRS) excitation takes place, which leads to a stronger enhancement of the Raman emission of MB. The signal amplification provided by the resonance effect (typically 2 orders of magnitude for MB), however, is expected to be independent of the substrate on which $\mathrm{MB}$ is deposited, so it is not supposed to influence our estimate of the SERS gain. On the other hand, by employing a pump laser at $785 \mathrm{~nm}$, for which no resonant Raman excitation takes place, the maximum gain increases, approaching the $10^{3}$ figure, which compares well with SERS substrates exploiting localized plasmon resonances of disconnected nanoparticles ${ }^{51,75}$ and lithographically made gratings. ${ }^{49,50}$ We can thus consider the present observations of relevance in view of molecular sensing applications since it is possible to reduce the NIR laser pump power (and in turn reduce sample damage and fluorescence background) while still keeping relevant the SERS gain in the near-infrared range of the spectrum. ${ }^{76,77}$

We stress that the discussed SERS gains could indeed be further increased by optimizing easily accessible experimental parameters. We can mention the increase of the grating period resulting in a red shift, beyond the onset of interband transitions, of the high-energy long-range hybrid SPP, which is characterized by a longer lifetime and a transverse electric field penetrating more deeply into the dielectric environment where probe molecules are found. ${ }^{67}$ Moreover, an optimization of the $\mathrm{Au}$ thin film thickness to find the ideal hybridization and photon-SPP coupling conditions could further maximize the SERS gain. Alternatively, the plasmonic response of our linearly graded nanogratings could be further tuned in view of plasmon-enhanced spectroscopies by, e.g., matching the pump laser frequency across the UV-to-NIR spectral range by growing conductive layers with different free carrier density. The SPP dispersion relation is in fact strongly affected by the metal plasma frequency: ${ }^{5,6}$ a blue shift toward the UV could be achieved by growing on top of the rippled dielectric template metal films with high density of free carriers such as $\mathrm{Ag}$ and $\mathrm{Al}$, while following a similar approach, one could imagine shifting the SPP excitation in the NIR-IR range by growing a thin film with a reduced free carrier density like, e.g., semiconductors with variable doping and $2 \mathrm{D}$ materials. ${ }^{78,79}$ The wide spectral tunability of the plasmonic self-organized nanogratings, combined with the possibility to easily scale up the low-cost and large-area fabrication method here described represents a crucial feature in view of a broad range of real-world applications aiming at photon harvesting and at advanced molecular sensing.

\section{CONCLUSIONS}

In this work, we demonstrate how a self-organized rippled soda lime glass template, prepared by an innovative wrinklingassisted ion-beam sputtering (IBS) method, can yield a largearea, subwavelength, quasi-1D blazed grating, enabling the unidirectional excitation of propagating hybrid surface plasmon polaritons (SPP) modes at the interface of a thin $\mathrm{Au}$ conductive film conformally grown on top of it. For this purpose, we engineered the IBS process to fabricate nanogratings with graded periodicity that act as tunable SPP couplers/launchers, thus facilitating the optimization of operating condition by, e.g., matching the pump laser frequency in plasmon-enhanced spectroscopies. A rippled $\mathrm{Au} /$ soda lime grating sample showed remarkable surfaceenhanced Raman scattering (SERS) activity in the detection of methylene blue molecules. We demonstrate how the SERS gain is strongly correlated with the electric field enhancement produced by the excitation of counter-propagating hybrid SPP modes by comparing SERS and co-localized microextinction measurements. The SERS gain observed employing a $785 \mathrm{~nm}$ pump laser is particularly striking, making the sample interesting for molecular and biosensing applications with low fluorescence background. Indeed, the SERS gain could be increased by further optimizing fabrication parameters such as the thin conductive film thickness and grating periodicity. The large-area self-organized SPP launching platform is very versatile since, by simply choosing the metal to conformally grow on top of the rippled glass template, it is possible to tailor the plasmonic response in a wide spectral range extending from the near-UV to the near-IR, and possibly beyond considering the use of semiconducting and $2 \mathrm{D}$ materials.

\section{ASSOCIATED CONTENT}

\section{Supporting Information}

The Supporting Information is available free of charge at https://pubs.acs.org/doi/10.1021/acsanm.0c01569.

Additional morphological and optical characterization data; self-correlation of the AFM topography, the black scale bar corresponds to $800 \mathrm{~nm}$ (Figure S1); sketch of the sample: red dots indicate the coordinates of different AFM measurements, while the blue arrows indicate the incoming Argon ion beam direction (left panel) (Figure S2); 2D auto-correlation of a AFM topography acquired on the Au/glass self-organized nanograting (Figure S3); transmission spectra acquired on the nanowires with polarization parallel to the long axis and calculated spectrum; spectra are acquired on different points (Figure S4); transmission spectra colocalized with SERS measurements acquired at different sample coordinates corresponding to the following grating periodicities: $171 \mathrm{~nm}$ (red curve), $198 \mathrm{~nm}$ (blue curve), $211 \mathrm{~nm}$ (cyan curve), $226 \mathrm{~nm}$ (yellow curve) (Figure S5) (PDF) 


\section{AUTHOR INFORMATION}

\section{Corresponding Author}

Francesco Buatier de Mongeot - Dipartimento di Fisica, Università di Genova, I-16146 Genova, Italy; 이이.org/ 0000-0002-8144-701X; Email: buatier@fisica.unige.it

\section{Authors}

Matteo Barelli - Dipartimento di Fisica, Università di Genova, I-16146 Genova, Italy; ○ orcid.org/0000-0002-3960-7921

Maria Caterina Giordano - Dipartimento di Fisica, Università di Genova, I-16146 Genova, Italy; 이 orcid.org/0000-00029757-4339

Pietro Giuseppe Gucciardi - CNR IPCF Istituto per i Processi Chimico-Fisici, I-98156 Messina, Italy; 이이.org/00000003-1826-9174

Complete contact information is available at:

https://pubs.acs.org/10.1021/acsanm.0c01569

\section{Notes}

The authors declare no competing financial interest.

\section{ACKNOWLEDGMENTS}

Financial support from Ministero dell'Università e della Ricerca Scientifica (MIUR) through the PRIN 2015 Grant 2015WTW7J3 and from Compagnia di San Paolo in the framework of Project ID ROL 9361 is gratefully acknowledged. F.B.d.M. thanks Roberto Chittofrati and Ennio Vigo for providing technical support.

\section{REFERENCES}

(1) Gramotnev, D. K.; Bozhevolnyi, S. I. Plasmonics beyond the Diffraction Limit. Nat. Photonics 2010, 4, 83-91.

(2) Lal, S.; Link, S.; Halas, N. J. Nano-Optics from Sensing to Waveguiding. Nat. Photonics 2007, 1, 641-648.

(3) Maier, S. A.; Atwater, H. A. Plasmonics: Localization and Guiding of Electromagnetic Energy in Metal/Dielectric Structures. J. Appl. Phys. 2005, 98, No. 011101.

(4) Anker, J. N.; Hall, W. P.; Lyandres, O.; Shah, N. C.; Zhao, J.; Van Duyne, R. P. Biosensing with Plasmonic Nanosensors. Nat. Mater. 2008, 7, 442-453.

(5) Maier, S. A. Plasmonics: Fundamentals and Applications; Springer: New York, 2007.

(6) Zayats, A. V.; Smolyaninov, I. I.; Maradudin, A. A. Nano-Optics of Surface Plasmon Polaritons. Phys. Rep. 2005, 408, 131-314.

(7) Low, T.; Chaves, A.; Caldwell, J. D.; Kumar, A.; Fang, N. X.; Avouris, P.; Heinz, T. F.; Guinea, F.; Martin-Moreno, L.; Koppens, F. Polaritons in Layered Two-Dimensional Materials. Nat. Mater. 2017, $16,182-194$.

(8) Grigorenko, A. N.; Polini, M.; Novoselov, K. S. Graphene Plasmonics. Nat. Photonics 2012, 6, 749-758.

(9) Smith, T. B.; Principi, A. Topological Surface-PlasmonPolaritons on Corrugated Metal-Dielectric Surfaces. 2020, arXiv:physics/2007.00025. arXiv.org e-Print archive. https://arxiv.org/abs/ 2007.00025

(10) Della Valle, G.; Longhi, S. Geometric Potential for Plasmon Polaritons on Curved Surfaces. J. Phys. B: At., Mol. Opt. Phys. 2010, 43, No. 051002.

(11) Han, Z.; Bozhevolnyi, S. I. Radiation Guiding with Surface Plasmon Polaritons. Rep. Prog. Phys. 2013, 76, No. 016402.

(12) Fang, Y.; Sun, M. Nanoplasmonic Waveguides: Towards Applications in Integrated Nanophotonic Circuits. Light: Sci. Appl. 2015, 4, e294.

(13) Wei, H.; Pan, D.; Zhang, S.; Li, Z.; Li, Q.; Liu, N.; Wang, W.; $\mathrm{Xu}, \mathrm{H}$. Plasmon Waveguiding in Nanowires. Chem. Rev. 2018, 118, $2882-2926$.
(14) Lu, H.; Gan, X.; Mao, D.; Zhao, J. Graphene-Supported Manipulation of Surface Plasmon Polaritons in Metallic Nanowaveguides. Photonics Res. 2017, 5, 162.

(15) Lu, H.; Gan, X.; Mao, D.; Jia, B.; Zhao, J. Flexibly Tunable High-Quality-Factor Induced Transparency in Plasmonic Systems. Sci. Rep. 2018, 8, No. 1558.

(16) Maccaferri, N.; Isoniemi, T.; Hinczewski, M.; Iarossi, M.; Strangi, G.; De Angelis, F. Designer Bloch Plasmon Polariton Dispersion in Grating-Coupled Hyperbolic Metamaterials. APL Photonics 2020, 5, No. 076109.

(17) Luo, X.; Tsai, D.; Gu, M.; Hong, M. Extraordinary Optical Fields in Nanostructures: From Sub-Diffraction-Limited Optics to Sensing and Energy Conversion. Chem. Soc. Rev. 2019, 48, 24582494.

(18) Fei, Z.; Rodin, A. S.; Andreev, G. O.; Bao, W.; McLeod, A. S.; Wagner, M.; Zhang, L. M.; Zhao, Z.; Thiemens, M.; Dominguez, G.; Fogler, M. M.; Neto, A. H. C.; Lau, C. N.; Keilmann, F.; Basov, D. N. Gate-Tuning of Graphene Plasmons Revealed by Infrared NanoImaging. Nature 2012, 487, 82-85.

(19) Ni, G. X.; McLeod, A. S.; Sun, Z.; Wang, L.; Xiong, L.; Post, K. W.; Sunku, S. S.; Jiang, B.-Y.; Hone, J.; Dean, C. R.; Fogler, M. M.; Basov, D. N. Fundamental Limits to Graphene Plasmonics. Nature 2018, 557, 530-533.

(20) Ni, G. X.; Wang, L.; Goldflam, M. D.; Wagner, M.; Fei, Z.; McLeod, A. S.; Liu, M. K.; Keilmann, F.; Özyilmaz, B.; Castro Neto, A. H.; Hone, J.; Fogler, M. M.; Basov, D. N. Ultrafast Optical Switching of Infrared Plasmon Polaritons in High-Mobility Graphene. Nat. Photonics 2016, 10, 244-247.

(21) Woessner, A.; Lundeberg, M. B.; Gao, Y.; Principi, A.; AlonsoGonzález, P.; Carrega, M.; Watanabe, K.; Taniguchi, T.; Vignale, G.; Polini, M.; Hone, J.; Hillenbrand, R.; Koppens, F. H. L. Highly Confined Low-Loss Plasmons in Graphene-Boron Nitride Heterostructures. Nat. Mater. 2015, 14, 421-425.

(22) Handbook of Enhanced Spectroscopy; Gucciardi, P. G.; de la Chapelle, M. L.; Lidgi-Guigui, N., Eds.; Pan Stanford Publishing: Singapore, 2016.

(23) Lee, J.; Crampton, K. T.; Tallarida, N.; Apkarian, V. A. Visualizing Vibrational Normal Modes of a Single Molecule with Atomically Confined Light. Nature 2019, 568, 78-82.

(24) Zhang, R.; Zhang, Y.; Dong, Z. C.; Jiang, S.; Zhang, C.; Chen, L. G.; Zhang, L.; Liao, Y.; Aizpurua, J.; Luo, Y.; Yang, J. L.; Hou, J. G. Chemical Mapping of a Single Molecule by Plasmon-Enhanced Raman Scattering. Nature 2013, 498, 82-86.

(25) Tallarida, N.; Rios, L.; Apkarian, V. A.; Lee, J. Isomerization of One Molecule Observed through Tip-Enhanced Raman Spectroscopy. Nano Lett. 2015, 15, 6386-6394.

(26) Wang, J.; Lin, W.; Cao, E.; Xu, X.; Liang, W.; Zhang, X. Surface Plasmon Resonance Sensors on Raman and Fluorescence Spectroscopy. Sensors 2017, 17, 2719.

(27) Kalachyova, Y.; Mares, D.; Lyutakov, O.; Kostejn, M.; Lapcak, L.; Svorčík, V. Surface Plasmon Polaritons on Silver Gratings for Optimal SERS Response. J. Phys. Chem. C 2015, 119, 9506-9512.

(28) Neff, H.; Oliveira, L. C.; Rodrigures, E. P.; Thirstrup, C.; Lima, A. M. N. Enhanced Directed Radiative Surface Plasmon Emission from Periodically Corrugated Noble Metal Films. Plasmonics 2018, 13, 2021-2027.

(29) Gillibert, R.; Sarkar, M.; Bryche, J.-F.; Yasukuni, R.; Moreau, J.; Besbes, M.; Barbillon, G.; Bartenlian, B.; Canva, M.; de la Chapelle, M. L. Directional Surface Enhanced Raman Scattering on Gold NanoGratings. Nanotechnology 2016, 27, No. 115202.

(30) Subramaniyam, N.; Shah, A.; Dreser, C.; Isomäki, A.; Fleischer, M.; Sopanen, M. Nonlinear Plasmonic Behavior of Nanohole Arrays in Thin Gold Films for Imaging Lipids. Appl. Phys. Lett. 2018, 112, No. 233109.

(31) Coca-López, N.; Hartmann, N. F.; Mancabelli, T.; Kraus, J.; Günther, S.; Comin, A.; Hartschuh, A. Remote Excitation and Detection of Surface-Enhanced Raman Scattering from Graphene. Nanoscale 2018, 10, 10498-10504. 
(32) Huang, Y.; Fang, Y.; Zhang, Z.; Zhu, L.; Sun, M. NanowireSupported Plasmonic Waveguide for Remote Excitation of SurfaceEnhanced Raman Scattering. Light: Sci. Appl. 2014, 3, e199.

(33) Xu, Y.; Bai, P.; Zhou, X.; Akimov, Y.; Png, C. E.; Ang, L.; Knoll, W.; Wu, L. Optical Refractive Index Sensors with Plasmonic and Photonic Structures: Promising and Inconvenient Truth. Adv. Opt. Mater. 2019, 7, No. 1801433.

(34) Homola, J.; Yee, S. S.; Gauglitz, G. Surface Plasmon Resonance Sensors: Review. Sens. Actuators, B 1999, 54, 3-15.

(35) Iqbal, T.; Afsheen, S. One Dimensional Plasmonic Grating: High Sensitive Biosensor. Plasmonics 2017, 12, 19-25.

(36) Bonse, J.; Rosenfeld, A.; Krüger, J. On the Role of Surface Plasmon Polaritons in the Formation of Laser-Induced Periodic Surface Structures upon Irradiation of Silicon by Femtosecond-Laser Pulses. J. Appl. Phys. 2009, 106, No. 104910.

(37) Gurevich, E. L.; Gurevich, S. V. Laser Induced Periodic Surface Structures Induced by Surface Plasmons Coupled via Roughness. Appl. Surf. Sci. 2014, 302, 118-123.

(38) Shimotsuma, Y.; Kazansky, P. G.; Qiu, J.; Hirao, K. SelfOrganized Nanogratings in Glass Irradiated by Ultrashort Light Pulses. Phys. Rev. Lett. 2003, 91, No. 247405.

(39) Wang, L.; Chen, Q.-D.; Cao, X.-W.; Buividas, R.; Wang, X.; Juodkazis, S.; Sun, H.-B. Plasmonic Nano-Printing: Large-Area Nanoscale Energy Deposition for Efficient Surface Texturing. Light: Sci. Appl. 2017, 6, e17112.

(40) Wang, Y.; Shen, L.; Wang, Y.; Hou, B.; Gibson, G. N.; Poudel, N.; Chen, J.; Shi, H.; Guignon, E.; Cady, N. C.; Page, W. D.; Pilar, A.; Dawlaty, J.; Cronin, S. B. Hot Electron-Driven Photocatalysis and Transient Absorption Spectroscopy in Plasmon Resonant Grating Structures. Faraday Discuss. 2019, 214, 325-339.

(41) Zhou, L.; Swearer, D. F.; Zhang, C.; Robatjazi, H.; Zhao, H.; Henderson, L.; Dong, L.; Christopher, P.; Carter, E. A.; Nordlander, P.; Halas, N. J. Quantifying Hot Carrier and Thermal Contributions in Plasmonic Photocatalysis. Science 2018, 362, 69-72.

(42) Clavero, C. Plasmon-Induced Hot-Electron Generation at Nanoparticle/Metal-Oxide Interfaces for Photovoltaic and Photocatalytic Devices. Nat. Photonics 2014, 8, 95-103.

(43) Knight, M. W.; Sobhani, H.; Nordlander, P.; Halas, N. J. Photodetection with Active Optical Antennas. Science 2011, 332, 702-704.

(44) Dan'ko, V.; Dmitruk, M.; Indutnyi, I.; Mamykin, S.; Myn’ko, V.; Shepeliavyi, P.; Lukaniuk, M.; Lytvyn, P. Au Gratings Fabricated by Interference Lithography for Experimental Study of Localized and Propagating Surface Plasmons. Nanoscale Res. Lett. 2017, 12, No. 190.

(45) Yu, C.-C.; Ho, K.-H.; Chen, H.-L.; Chuang, S.-Y.; Tseng, S.-C.; $\mathrm{Su}$, W.-F. Using the Nanoimprint-in-Metal Method to Prepare Corrugated Metal Structures for Plasmonic Biosensors through Both Surface Plasmon Resonance and Index-Matching Effects. Biosens. Bioelectron. 2012, 33, 267-273.

(46) Gao, L.; Lemarchand, F.; Lequime, M. Comparison of Different Dispersion Models for Single Layer Optical Thin Film Index Determination. Thin Solid Films 2011, 520, 501-509.

(47) Safar, W.; Lequeux, M.; Solard, J.; Fischer, A. P. A.; Felidj, N.; Gucciardi, P. G.; Edely, M.; de la Chapelle, M. L. Gold Nanocylinders on Gold Film as a Multi-Spectral SERS Substrate. Nanomaterials 2020, 10, 927.

(48) Giordano, M. C.; Buatier de Mongeot, F. Anisotropic Nanoscale Wrinkling in Solid-State Substrates. Adv. Mater. 2018, 30, No. e1801840.

(49) Cottat, M.; D’Andrea, C.; Yasukuni, R.; Malashikhina, N.; Grinyte, R.; Lidgi-Guigui, N.; Fazio, B.; Sutton, A.; Oudar, O.; Charnaux, N.; Pavlov, V.; Toma, A.; Di Fabrizio, E.; Gucciardi, P. G.; de la Chapelle, M. L. High Sensitivity, High Selectivity SERS Detection of MnSOD Using Optical Nanoantennas Functionalized with Aptamers. J. Phys. Chem. C 2015, 119, 15532-15540.

(50) Kessentini, S.; Barchiesi, D.; D’Andrea, C.; Toma, A.; Guillot, N.; Di Fabrizio, E.; Fazio, B.; Maragó, O. M.; Gucciardi, P. G.; de la Chapelle, M. L. Gold Dimer Nanoantenna with Slanted Gap for
Tunable LSPR and Improved SERS. J. Phys. Chem. C 2014, 118, 3209-3219.

(51) D’Andrea, C.; Fazio, B.; Gucciardi, P. G.; Giordano, M. C.; Martella, C.; Chiappe, D.; Toma, A.; Buatier de Mongeot, F.; Tantussi, F.; Vasanthakumar, P.; Fuso, F.; Allegrini, M. SERS Enhancement and Field Confinement in Nanosensors Based on Self-Organized Gold Nanowires Produced by Ion-Beam Sputtering. J. Phys. Chem. C 2014, 118, 8571-8580.

(52) Lamberti, A.; Virga, A.; Angelini, A.; Ricci, A.; Descrovi, E.; Cocuzza, M.; Giorgis, F. Metal-Elastomer Nanostructures for Tunable SERS and Easy Microfluidic Integration. RSC Adv. 2015, 5, 4404-4410.

(53) Repetto, D.; Giordano, M. C.; Foti, A.; Gucciardi, P. G.; Mennucci, C.; Buatier de Mongeot, F. SERS Amplification by UltraDense Plasmonic Arrays on Self-Organized PDMS Templates. Appl. Surf. Sci. 2018, 446, 83-91.

(54) Abbasi, A.; de Oliveira, T. T. S.; Bothun, G. D.; Bose, A. Carbon Black Templated Gold Nanoparticles for Detection of a Broad Spectrum of Analytes by Surface-Enhanced Raman Scattering. ACS Appl. Nano Mater. 2020, 3, 2605-2613.

(55) Horcas, I.; Fernández, R.; Gómez-Rodríguez, J. M.; Colchero, J.; Gómez-Herrero, J.; Baro, A. M. WSXM: A Software for Scanning Probe Microscopy and a Tool for Nanotechnology. Rev. Sci. Instrum. 2007, 78, No. 013705.

(56) Nečas, D.; Klapetek, P. Gwyddion: An Open-Source Software for SPM Data Analysis. Open Physics 2012, 10, No. 99.

(57) Giordano, M. C.; Repetto, D.; Mennucci, C.; Carrara, A.; Buatier de Mongeot, F. Template-Assisted Growth of Transparent Plasmonic Nanowire Electrodes. Nanotechnology 2016, 27, No. 495201.

(58) Mennucci, C.; Del Sorbo, S.; Pirotta, S.; Galli, M.; Andreani, L. C.; Martella, C.; Giordano, M. C.; Buatier de Mongeot, F. Light Scattering Properties of Self-Organized Nanostructured Substrates for Thin-Film Solar Cells. Nanotechnology 2018, 29, No. 355301.

(59) Belardini, A.; Pannone, F.; Leahu, G.; Larciprete, M. C.; Centini, M.; Sibilia, C.; Martella, C.; Giordano, M. C.; Chiappe, D.; Buatier de Mongeot, F. Evidence of Anomalous Refraction of SelfAssembled Curved Gold Nanowires. Appl. Phys. Lett. 2012, 100, No. 251109.

(60) Giordano, M. C.; Longhi, S.; Barelli, M.; Mazzanti, A.; Buatier de Mongeot, F.; Della Valle, G. Plasmon Hybridization Engineering in Self-Organized Anisotropic Metasurfaces. Nano Res. 2018, 11, 39433956.

(61) Barelli, M.; Mazzanti, A.; Giordano, M. C.; Della Valle, G.; Buatier de Mongeot, F. Color Routing via Cross-Polarized Detuned Plasmonic Nanoantennas in Large Area Metasurfaces. Nano Lett. 2020, No. 4121.

(62) Giordano, M. C.; Tzschoppe, M.; Barelli, M.; Vogt, J.; Huck, C.; Canepa, F.; Pucci, A.; Buatier de Mongeot, F. Self-Organized Nanorod Arrays for Large-Area Surface-Enhanced Infrared Absorption. ACS Appl. Mater. Interfaces 2020, 12, 11155-11162.

(63) Yakubovsky, D. I.; Arsenin, A. V.; Stebunov, Y. V.; Fedyanin, D.; Yu; Volkov, V. S. Optical Constants and Structural Properties of Thin Gold Films. Opt. Express 2017, 25, 25574.

(64) Kossoy, A.; Merk, V.; Simakov, D.; Leosson, K.; Kéna-Cohen, S.; Maier, S. A. Optical and Structural Properties of Ultra-Thin Gold Films. Adv. Opt. Mater. 2015, 3, 71-77.

(65) Park, J. H.; Ambwani, P.; Manno, M.; Lindquist, N. C.; Nagpal, P.; Oh, S.-H.; Leighton, C.; Norris, D. J. Single-Crystalline Silver Films for Plasmonics. Adv. Mater. 2012, 24, 3988-3992.

(66) Burke, J. J.; Stegeman, G. I.; Tamir, T. Surface-Polariton-like Waves Guided by Thin, Lossy Metal Films. Phys. Rev. B 1986, 33, 5186-5201

(67) Berini, P. Long-Range Surface Plasmon Polaritons. Adv. Opt. Photonics 2009, 1, 484.

(68) Sarid, D. Long-Range Surface-Plasma Waves on Very Thin Metal Films. Phys. Rev. Lett. 1981, 47, 1927-1930. 
(69) Inagaki, T.; Motosuga, M.; Arakawa, E. T.; Goudonnet, J. P. Coupled Surface Plasmons in Periodically Corrugated Thin Silver Films. Phys. Rev. B 1985, 32, 6238-6245.

(70) Chen, Z.; Hooper, I. R.; Sambles, J. R. Coupled Surface Plasmons on Thin Silver Gratings. J. Opt. A: Pure Appl. Opt. 2008, 10, No. 015007.

(71) Chen, Z.; Hooper, I. R.; Sambles, J. R. Strongly Coupled Surface Plasmons on Thin Shallow Metallic Gratings. Phys. Rev. B 2008, 77, No. 2135.

(72) Barnes, W. L.; Preist, T. W.; Kitson, S. C.; Sambles, J. R. Physical Origin of Photonic Energy Gaps in the Propagation of Surface Plasmons on Gratings. Phys. Rev. B 1996, 54, 6227-6244.

(73) Hooper, I. R.; Sambles, J. R. Coupled Surface Plasmon Polaritons on Thin Metal Slabs Corrugated on Both Surfaces. Phys. Rev. B 2004, 70, No. 665.

(74) D’Andrea, C.; Bochterle, J.; Toma, A.; Huck, C.; Neubrech, F.; Messina, E.; Fazio, B.; Maragò, O. M.; Di Fabrizio, E.; de La Chapelle, M. L.; Gucciardi, P. G.; Pucci, A. Optical Nanoantennas for Multiband Surface-Enhanced Infrared and Raman Spectroscopy. ACS Nano 2013, 7, 3522-3531.

(75) Fazio, B.; D’Andrea, C.; Bonaccorso, F.; Irrera, A.; Calogero, G.; Vasi, C.; Gucciardi, P. G.; Allegrini, M.; Toma, A.; Chiappe, D.; Martella, C.; Buatier de Mongeot, F. Re-Radiation Enhancement in Polarized Surface-Enhanced Resonant Raman Scattering of Randomly Oriented Molecules on Self-Organized Gold Nanowires. ACS Nano 2011, 5, 5945-5956.

(76) Nie, S. Probing Single Molecules and Single Nanoparticles by Surface-Enhanced Raman Scattering. Science 1997, 275, 1102-1106.

(77) Barelli, M.; Repetto, D.; Buatier de Mongeot, F. Infrared Plasmonics via Self-Organized Anisotropic Wrinkling of Au/PDMS Nanoarrays. ACS Appl. Polym. Mater. 2019, 1, 1334-1340.

(78) Taliercio, T.; Biagioni, P. Semiconductor Infrared Plasmonics. Nanophotonics 2019, 8, 949-990.

(79) Zhu, X.; Wang, W.; Yan, W.; Larsen, M. B.; Bøggild, P.; Pedersen, T. G.; Xiao, S.; Zi, J.; Mortensen, N. A. Plasmon-Phonon Coupling in Large-Area Graphene Dot and Antidot Arrays Fabricated by Nanosphere Lithography. Nano Lett. 2014, 14, 2907-2913. 